\title{
STOP-Bang Questionnaire in Patients with Rapid Eye Movement Sleep Behavior Disorder
}

\author{
Ki-Hwan Ji, MD, Mi-Ri Kang, MD \\ Department of Neurology, Busan Paik Hospital, Inje University College of Medicine, Busan, Korea
}

Background and Objective The snoring, tiredness, observed apnea, and high blood pressurebody mass index, age, neck circumference, and gender (STOP-Bang) questionnaire is known as a simple but useful tool for the diagnosis of high-risk obstructive sleep apnea (OSA). However, the utility of STOP-Bang questionnaire in rapid eye movement (REM) sleep behavior disorder (RBD) populations is not validated. This study aimed to determine the diagnostic value of the STOP-Bang questionnaire in patients with RBD at high risk for OSA.

Methods We collected data from 65 consecutive patients who were diagnosed with RBD in a tertiary sleep center ( 20 women; mean age, $64.3 \pm 12.5$ years). All the patients visited sleep center with complaints of abnormal behavior during sleep, and underwent testing with STOP-Bang questionnaire and polysomnography. The diagnosis of RBD was based on the International Classification of Sleep Disorders, second edition. We diagnosed OSA when apnea-hypopnea index (AHI) was at least $5 / \mathrm{h}$. The receiver operating characteristic (ROC) curves were plotted.

Results The mean AHI was $18.2 \pm 16.5 / \mathrm{h}$, and $75.4 \%(\mathrm{n}=49)$ had an AHI $\geq 5$. The STOP-Bang (threshold $\geq 3$ ) identified $70.7 \%$ of patients as high risk for OSA, and sensitivity, specificity, positive and negative predictive values were $81.6,62.5,87$, and $52.6 \%$, respectively. The area under the ROC curve (AUC) was $0.79(\mathrm{p}<0.001)$. The STOP (threshold $\geq 2$ ) identified $70.7 \%$ of patients at high risk for OSA, and sensitivity, specificity, positive and negative predictive values were 75.5, 87.5, 94.9, and $53.8 \%$, respectively. The AUC was $0.86(\mathrm{p}<0.001)$. A pairwise comparison of ROC curve between STOP-Bang and STOP was insignificant $(\mathrm{p}=0.145)$.

Conclusions In RBD population, the STOP-Bang or STOP questionnaire is a useful screening tool to identify patients at high risk for OSA.

Sleep Med Res 2017;8(2):102-106

Key Words REM sleep behavior disorder, Obstructive sleep apnea, Screening questionnaire.

\section{INTRODUCTION}

Received: November 22, 2017

Revised: December 12, 2017

Accepted: December 15, 2017

Correspondence

Ki-Hwan Ji, MD

Department of Neurology,

Busan Paik Hospital, Inje University

College of Medicine,

75 Bokji-ro, Busanjin-gu,

Busan 47392, Korea

Tel +82-51-890-8613

Fax +82-51-890-6130

E-mail kihwanji@gmail.com

ORCID

Ki-Hwan Ji

https://orcid.org/0000-0002-5371-5398

Mi-Ri Kang

https://orcid.org/0000-0002-9833-023X
Rapid eye movement (REM) sleep behavior disorder (RBD) is characterized by dream-enacting behavior during REM sleep, accompanied by loss of normal REM sleep muscle atonia. ${ }^{1}$ The estimated prevalence of RBD is in the range of $0.5 \%$ to $2 \% .^{2}$ Loss of REM sleep atonia is diagnostic for RBD, although probable RBD may be diagnosed on clinical grounds. Obstructive sleep apnea (OSA) is also prevalent in adults and caused by repetitive complete or partial obstruction of the upper airway during sleep. OSA, potentially reversible with continuous positive airway pressure (CPAP) therapy is one of the risk factors for diabetes, hypertension, arrhythmia, cardio and cerebrovascular events. ${ }^{3}$ OSA and RBD frequently co-occur, mainly due to shared risk factors including age and male gender. ${ }^{4,5}$ The STOP-Bang questionnaire is a simple self-reported screening tool comprising snoring, tiredness, observed apnea, and high blood pressure (STOP) and Bang demographic items [body mass index (BMI), age, neck circumference, and gender]. ${ }^{6}$ The STOP-Bang questionnaire has been widely used to recognize patients at high- 
risk for OSA. ${ }^{7}$ However, recent evidence suggests obesity and snoring may be less critical diagnostic markers of OSA in RBD patients manifesting lower BMI and less snoring than OSA patients without RBD. ${ }^{4}$ Therefore, the STOP-Bang questionnaire may be less useful in screening the OSA associated with RBD patients than in general population. However, the role of STOPBang questionnaire in RBD populations has not been studied until now. We hypothesized that the STOP-Bang questionnaire has low diagnostic ability in identifying RBD patients with high risk for OSA. This study sought to determine the diagnostic value of the STOP-Bang questionnaire in recognizing patients with RBD at high risk for OSA.

\section{METHODS}

Sixty-five consecutive patients who were diagnosed with RBD in a single tertiary sleep center from January 2012 to June 2017 were included. All the patients visited sleep center with the main complaints of abnormal or dream-enacting behaviors during sleep. Patients were diagnosed with RBD based on clinical interviews and polysomnography (PSG). All the patients had completed STOP-Bang questionnaire, Epworth Sleepiness Scale, and Beck Depression Inventory before PSG as routine practices. The STOP-Bang questionnaire includes four yes/no questions evaluating loud snoring, daytime fatigue, apnea observed during sleep by another person, and a diagnosis or treatment for high blood pressure indicative of STOP. According to original STOP-Bang questionnaire, demographic items were deemed positive when BMI exceeded $35 \mathrm{~kg} / \mathrm{m}^{2}$, age was greater than 50 years, neck circumference was more than $40 \mathrm{~cm}$ measured below the laryngeal prominence, and male gender. ${ }^{6}$ Patients answering two or more items on STOP questionnaire and three or more items on STOP-Bang questionnaire were considered as high-risk candidates for OSA. ${ }^{6}$ PSG was performed according to the previous recommendations ${ }^{8}$ and done using RemLogic (Embla System, Denver, CO, USA). The diagnosis of RBD was in accordance with the International Classification of Sleep Disorders, second edition. ${ }^{1}$ We diagnosed OSA when the apneahypopnea index $(\mathrm{AHI}) \geq 5 / \mathrm{h}$. This study was approved by the Institutional Review Board of the Inje University Busan Paik Hospital (IRB No. 17-0108). The Institutional Review Board granted a waiver of informed consent because it was retrospective chart review study.

\section{Statistical Analysis}

We presented the demographic data with descriptive statistics. Continuous data were presented as means ( \pm standard deviation) and compared with Student t-test or Mann-Whitney $\mathrm{U}$ test according to the data distribution. Categorical data were presented as frequencies, and compared with Chi-square or Fisher's exact test. We determined the sensitivity, specificity, positive predictive value, and negative predictive value for highrisk OSA using STOP-Bang questionnaire. The area under the receiver operating characteristic (ROC) curve (AUC) was plotted for high risk for the STOP-Bang questionnaire, and the cutoff values were calculated. In determining optimal cut-off value, Youden index, which uses the maximum of vertical distance of ROC curve from the point on diagonal line, was used. We compared the STOP-Bang and STOP questionnaire. We used Stata (StataCorp, 2015, Stata Statistical Software: Release 14, College Station, TX, USA) for statistical analysis. The null hypothesis was rejected at $\mathrm{p}<0.05$.

\section{RESULTS}

\section{Demographics and PSG Variables}

A total of 65 patients with RBD included 56 with idiopathic RBD and 9 with symptomatic RBD (Parkinson disease, 5; multiple system atrophy, 4). The male-to-female ratio was 2.3:1 ( $\mathrm{n}=$ 45 vs. 20 ), and the mean age was $64.3 \pm 12.5$ years. The mean AHI was $18.2 \pm 16.5 / \mathrm{h}$, and $75.4 \%(\mathrm{n}=49)$ of the patients had OSA defined as an $\mathrm{AHI} \geq 5 / \mathrm{h}$. The prevalence of moderate to severe OSA (AHI $\geq 15 / \mathrm{h}$ ) was $44.6 \%$. Twenty out of $25(80 \%)$ female RBD patients had OSA, and 29 out of 40 (72.5\%) male RBD patients had OSA. The age, BMI, and neck circumference were no significant difference between RBD with OSA and without OSA. The arousal index and N1 proportion were significantly high in RBD with OSA ( $p=0.005$ and $p=0.008$, respectively). The demographics and PSG variables are summarized in Table 1.

\section{The STOP-Bang Questionnaire}

Among the RBD cases, 32 (49.2\%) marked 'yes' to snoring question, 28 (43.1\%) for tiredness, 24 (36.9\%) for observed apnea, and 26 (40\%) for high blood pressure. Each proportion of positive STOP item was significantly higher in RBD with OSA than RBD without OSA. No single subject manifested BMI over $30 \mathrm{~kg} / \mathrm{m}^{2}$, and five $(7.7 \%)$ were aged under 50 years. Fourteen (21.5\%) patients, all male, had neck circumference exceeding 40 $\mathrm{cm}$. The STOP-Bang (threshold $\geq 3$ ) identified $70.7 \%$ of patients as high risk for OSA, and sensitivity, specificity, positive and negative predictive values were $81.6,62.5,87$, and $52.6 \%$, respectively. The AUC was $0.79(\mathrm{p}<0.001)$. The STOP (threshold $\geq 2$ ) identified $70.7 \%$ of patients at high risk for OSA, and sensitivity, specificity, positive and negative predictive values were 75.5, $87.5,94.9$, and $53.8 \%$, respectively. The AUC was 0.86 ( $p<0.001)$. A pairwise comparison of ROC curve between STOP-Bang and STOP was insignificant ( $\mathrm{p}=0.145$ ) (Fig. 1).

In moderate to severe OSA (AHI $\geq 15 / h$ ), the STOP-Bang (threshold $\geq 3$ ) had sensitivity, specificity, positive and negative predictive values of $82.8,36.1,51.1$, and $72.2 \%$, respectively. The AUC was $0.64(\mathrm{p}=0.035)$. The STOP (threshold $\geq 2)$ had 
Table 1. Patient demographics and PSG findings of REM sleep behavior with and without OSA

\begin{tabular}{|c|c|c|c|c|}
\hline Variables & Total & RBD without OSA & RBD with OSA & p-value \\
\hline Number of patients & 65 & 16 & 49 & \\
\hline Age at diagnosis (year) & $64.3 \pm 12.5$ & $60.2 \pm 15.5$ & $65.6 \pm 11.2$ & 0.237 \\
\hline Female, n (\%) & $25(38)$ & $5(31)$ & $20(41)$ & 0.495 \\
\hline $\mathrm{BMI}\left(\mathrm{kg} / \mathrm{m}^{2}\right)$ & $23.6 \pm 2.5$ & $23.3 \pm 2.1$ & $23.8 \pm 2.6$ & 0.469 \\
\hline Neck circumferences $(\mathrm{cm})$ & $36.9 \pm 3.1$ & $36.4 \pm 3.5$ & $37.2 \pm 3.0$ & 0.414 \\
\hline ESS & $7.2 \pm 5.7$ & $8.3 \pm 6.1$ & $6.9 \pm 5.5$ & 0.459 \\
\hline BDI & $15.3 \pm 9.7$ & $16.1 \pm 8.9$ & $15.0 \pm 10.1$ & 0.706 \\
\hline \multicolumn{5}{|l|}{ PSG results } \\
\hline Total sleep time ( $\mathrm{min})$ & $378.3 \pm 82.4$ & $387.6 \pm 82.9$ & $375.4 \pm 82.8$ & 0.621 \\
\hline Sleep latency (min) & $16.9 \pm 26.7$ & $13.8 \pm 15.6$ & $17.9 \pm 30$ & 0.451 \\
\hline REM latency (min) & $93.2 \pm 66.6$ & $109.6 \pm 72.9$ & $87.5 \pm 64$ & 0.218 \\
\hline Sleep efficiency (\%) & $79.7 \pm 12.5$ & $78.2 \pm 15.6$ & $80.2 \pm 11.5$ & 0.939 \\
\hline Arousal index $(/ \mathrm{hr})$ & $21.8 \pm 11.4$ & $15.8 \pm 10.1$ & $23.8 \pm 11.2$ & $0.005^{*}$ \\
\hline $\mathrm{AHI}(/ \mathrm{hr})$ & $18.2 \pm 16.5$ & $2.4 \pm 1.5$ & $23.4 \pm 15.9$ & $<0.001^{\dagger}$ \\
\hline PLMI (/hr) & $22.7 \pm 36.1$ & $21.1 \pm 36.7$ & $23.2 \pm 36.3$ & 0.876 \\
\hline \multicolumn{5}{|l|}{ Sleep architecture (\%) } \\
\hline $\mathrm{N} 1$ & $30.7 \pm 21.3$ & $20.4 \pm 10.2$ & $34.1 \pm 22.9$ & $0.008^{*}$ \\
\hline $\mathrm{N} 2$ & $50.8 \pm 15.3$ & $59.3 \pm 10.5$ & $48 \pm 15.7$ & $0.019^{*}$ \\
\hline N3 & $0.4 \pm 1.3$ & $0.4 \pm 1.2$ & $0.4 \pm 1.3$ & 0.631 \\
\hline REM & $20.1 \pm 8.6$ & $19.9 \pm 8.4$ & $20.1 \pm 8.8$ & 0.855 \\
\hline \multicolumn{5}{|l|}{ Questionnaire } \\
\hline STOP-Bang & $3.5 \pm 1.5$ & $2.4 \pm 1.0$ & $3.8 \pm 1.2$ & $<0.001^{\dagger}$ \\
\hline STOP & $1.8 \pm 1.3$ & $0.7 \pm 0.7$ & $2.2 \pm 1.2$ & $<0.001^{\dagger}$ \\
\hline Snoring, n (\%) & & $3(19)$ & $32(49)$ & $0.002^{*}$ \\
\hline Tiredness, $\mathrm{n}(\%)$ & & $5(31)$ & $28(43)$ & 0.089 \\
\hline Observed apnea, n (\%) & & 0 & $24(37)$ & $<0.001^{\dagger}$ \\
\hline High blood pressure, n (\%) & & $3(19)$ & $26(40)$ & $0.021^{*}$ \\
\hline
\end{tabular}

${ }^{*} \mathrm{p}<0.05 .{ }^{\dagger} \mathrm{p}<0.001$.

RBD: REM sleep behavior disorder, BMI: body mass index, ESS: Epworth sleepiness scale, BDI: beck depression inventory, AHI: apnea-hypopnea index, PLMI: periodic limb movement index, REM: rapid eye movement, PSG: polysomnography, OSA: obstructive sleep apnea, REM: rapid eye movement, STOP-Bang: snoring, tiredness, observed apnea, and high blood pressure-body mass index, age, neck circumference, and gender.

sensitivity, specificity, positive and negative predictive values of $72.4,55.6,56.8$, and $71.4 \%$, respectively. The AUC was $0.67(\mathrm{p}=$ 0.007). The comparison of ROC curve between STOP-Bang and STOP was insignificant ( $\mathrm{p}=0.359$ ) (Fig. 1). The cut-off values of STOP-Bang (threshold $\geq 3$ ) and STOP (threshold $\geq 2$ ) questionnaire were same whether based on an $\mathrm{AHI} \geq 5 / \mathrm{h}$ or $\mathrm{AHI} \geq$ $15 /$ h.

\section{DISCUSSION}

We evaluated the diagnostic value of STOP-Bang questionnaire in Korean RBD patients in a single tertiary sleep center. The prevalence of OSA was $75.4 \%$ for an $\mathrm{AHI} \geq 5 / \mathrm{h}$. The mean
BMI was $23.6 \mathrm{~kg} / \mathrm{m}^{2}$. The STOP-Bang had a sensitivity of $81.6 \%$ and STOP had a specificity of $87.5 \%$, for identification of RBD patients at high risk for OSA $(A H I \geq 5 / h)$. In moderate to severe OSA (AHI $\geq 15 / h$ ), the STOP-Bang had a sensitivity of $82.8 \%$ and STOP had a specificity of $55.6 \%$. The level of sensitivity of STOP-Bang and STOP for identifying high-risk OSA (AHI $\geq 5 / h$ and $A H I \geq 15 / h$ ) in RBD patients was lower, but specificity was higher than those of the previous study. In literature, the STOP-Bang questionnaire had a high sensitivity of $97 \%$, but low specificity of $18.6 \%$ for identifying Korean OSA patients. ${ }^{7}$ Positive and negative predictive values were $85.9 \%$ and $54.6 \%$, respectively. ${ }^{9}$

As mentioned previously, OSA and RBD share risk factors of age and male gender. ${ }^{4}$ RBD is prevalent in males and typically 

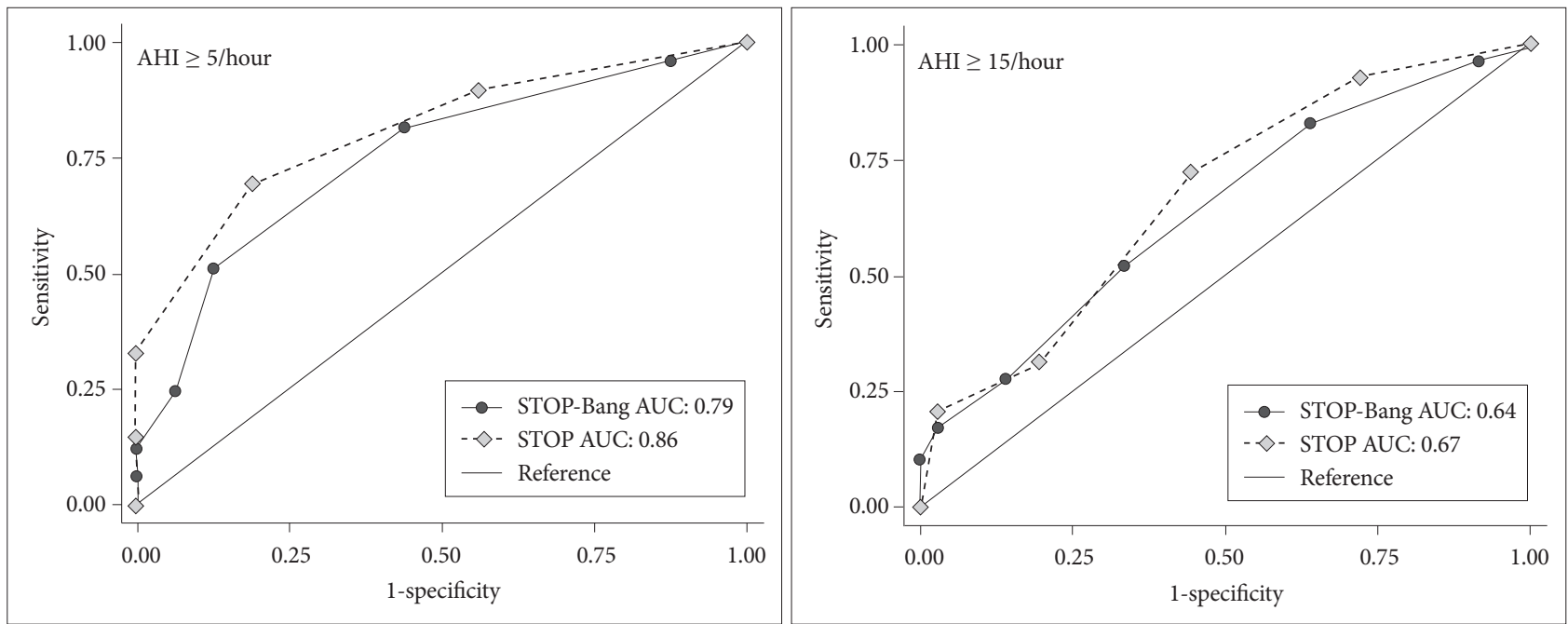

Fig. 1. ROC curves for the STOP-Bang and STOP with an AHI cutoff $\geq 5 /$ hour and AHI cutoff $\geq 15 / h$. The ROC curve is a graphical plot displaying the diagnostic ability of a binary classifier. The overall accuracy of the ROC curve is expressed as the AUC, which ranges between 0 and 1. The diagnostic accuracy of the test is better, when AUC is closer to 1. The AUC is given in the legend of each graph. ROC: receiver operating characteristic, AHI: apnea-hypopnea index, AUC: area under the ROC curve, STOP-Bang: snoring, tiredness, observed apnea, and high blood pressure-body mass index, age, neck circumference, and gender.

occurs in $40-70$ year-old patients. ${ }^{10}$ Despite the high prevalence of OSA detected in both genders (male, $72.5 \%$; female, $80 \%$ ) and association with old age ( $92.3 \%$ were over 50 years) in this study, no additional information was available even though gender and age were used to predict OSA in RBD patients by STOPBang questionnaire. The average BMI $\left(23.6 \mathrm{~kg} / \mathrm{m}^{2}\right)$ in this study with a BMI cutoff of $35 \mathrm{~kg} / \mathrm{m}^{2}$ was also non-discriminatory, because all RBD patients showed BMI less than $30 \mathrm{~kg} / \mathrm{m}^{2}$. These results are compatible with findings that obesity was less critical as a diagnostic marker of OSA in RBD patients with BMI lower than in OSA patients without RBD. ${ }^{4} \mathrm{~A}$ comparison of ROC curve between STOP-Bang and STOP was also insignificant. Therefore, addition of the Bang questionnaire to STOP did not improve the sensitivity.

The ICSD-2 diagnostic criteria for RBD warrant the exclusion of other medical or neurological conditions, which may be associated with RBD-mimics. ${ }^{1}$ Severe OSA mimics the symptoms of RBD, which could be relieved by CPAP. ${ }^{11}$ Although the impact of comorbid OSA in RBD patients with cardiovascular and neurodegenerative progression is still controversial, ${ }^{5}$ a recent study showed that OSA accompanied with increased non-motor symptoms, including cognitive, urinary, gastrointestinal dysfunction, and daytime sleepiness. ${ }^{4}$ Furthermore, one of the first-line medications, clonazepam may worsen sleep apnea even at low doses in RBD patients. ${ }^{12}$ Regarding removal of sleep-related injury or injurious behaviors, RBD comorbid with OSA is also associated with suboptimal response to clonazepam treatment. ${ }^{13}$

The standard diagnostic tool for OSA is PSG, despite the tedious and expensive features and poor accessibility in certain jurisdictions. In real practice, all probable RBD patients are not diagnosed with PSG. Thus the use of STOP-Bang in managing probable RBD patients may have the following meanings in clinical practice. First, when PSG is planned, the risk of having OSA can be adopted as a factor to the priority of the PSG. Second, both patient and sleep technician are informed of high risk of having OSA in advance that can easily proceed to a split-night study or CPAP treatment if necessary. Third, probable RBD patients unable to undergo PSG may have different drug choice depending on risk of OSA comorbidity. The use of clonazepam may be avoided. Finally, a physician can continuously monitor OSA risk of RBD patients using STOP-Bang and improve the quality of treatment. Therefore, STOP-Bang, a simple and easy screening tool for high-risk OSA may be useful in managing RBD patients in various clinical settings.

Our study has several limitations. Our subjects were limited to RBD patients who visited a single tertiary sleep center. Sleep clinic populations are diverse, and patients vary across different clinics. Therefore, our current findings cannot be generalized. Further, in this retrospective study, we failed to assess the testretest reliability of the questionnaire. In previous studies, the agreement of the STOP questionnaire was across a high range of $88-96 \%{ }^{6,14}$

In conclusion, we demonstrated that the STOP-Bang or STOP questionnaire represents a useful screening tool for identification of Korean RBD patients at high risk for OSA.

\section{Acknowledgments}

This work was supported by grant from Inje University, 2011.

\section{Conflicts of Interest}

The authors have no financial conflicts of interest.

\section{Authors' Contribution}

Conceptualization, investigation, data mining and analysis, interpretation, writing_original draft, review \& editing: Ji KH. Writing_-review \& editing: Kang MR. 


\section{REFERENCES}

1. AASM. International classification of sleep disorders: diagnostic and coding manual. Westchester: American Academy of Sleep Medicine 2005.

2. St Louis EK, Boeve BF. REM sleep behavior disorder: diagnosis, clinical implications, and future directions. Mayo Clin Proc 2017;92:1723-36.

3. Khayat R, Pleister A. Consequences of obstructive sleep apnea: cardiovascular risk of obstructive sleep apnea and whether continuous positive airway pressure reduces that risk. Sleep Med Clin 2016;11:273-86.

4. Bugalho P, Mendonça M, Barbosa R, Salavisa M. The influence of sleep disordered breathing in REM sleep behavior disorder. Sleep Med 2017;37:210-5

5. Zhang J, Li SX, Lam SP, Wing YK. REM sleep behavior disorder and obstructive sleep apnea: does one "evil" make the other less or more "evil"? Sleep Med 2017;37:216-7.

6. Chung F, Yegneswaran B, Liao P, Chung SA, Vairavanathan S, Islam S, et al. STOP questionnaire: a tool to screen patients for obstructive sleep apnea. Anesthesiology 2008;108:812-21.

7. Nagappa M, Liao P, Wong J, Auckley D, Ramachandran SK, Memtsoudis S, et al. Validation of the STOP-Bang Questionnaire as a screening tool for obstructive sleep apnea among different populations: a systematic review and meta-analysis. PLoS One 2015;10:e0143697.
8. Iber C, Ancoli-Israel S, Chesson AL, Quan SF. The AASM manual for the scoring of sleep and associated events: rules, terminology and technical specifications. Westchester: American Academy of Sleep Medicine 2007.

9. Lee SA, Paek JH, Han SH, Ryu HU. The utility of a Korean version of the REM sleep behavior disorder screening questionnaire in patients with obstructive sleep apnea. J Neurol Sci 2015;358:328-32.

10. Boeve BF. REM sleep behavior disorder: updated review of the core features, the REM sleep behavior disorder-neurodegenerative disease association, evolving concepts, controversies, and future directions. Ann N Y Acad Sci 2010;1184:15-54.

11. Iranzo A, Santamaría J. Severe obstructive sleep apnea/hypopnea mimicking REM sleep behavior disorder. Sleep 2005;28:203-6.

12. Schuld A, Kraus T, Haack M, Hinze-Selch D, Pollmächer T. Obstructive sleep apnea syndrome induced by clonazepam in a narcoleptic patient with REM-sleep-behavior disorder. I Sleep Res 1999;8:321-2.

13. Li SX, Lam SP, Zhang J, Yu MW, Chan JW, Liu Y, et al. A prospective, naturalistic follow-up study of treatment outcomes with clonazepam in rapid eye movement sleep behavior disorder. Sleep Med 2016;21:114-20.

14. Sadeghniiat-Haghighi K, Montazeri A, Khajeh-Mehrizi A, Ghajarzadeh M, Alemohammad ZB, Aminian O, et al. The STOP-BANG questionnaire: reliability and validity of the Persian version in sleep clinic population. Qual Life Res 2015;24:2025-30. 